\title{
OBTENCION DE PULPA KRAFT A PARTIR DE \\ Pinus patula, Pinus taeda y Pinus elliottii \\ CRECIDOS EN CHILE
}

Sandra Rodriguez $\mathrm{S}^{(*)}$

Marco Torres U(**)

\section{RESUMEN}

Este trabajo tuvo como objetivo estudiar el comportamiento de las especies Pinus patula, Pinus taeda y Pinus elliottii frente al proceso kraft. Las muestras usadas correspondieron a árboles de raleo del Arboretum, ubicado en el Campus Isla Teja de la Universidad Austral de Chile.

Se determinó la composición química de las maderas y la densidad de las astillas. Se realizaron ensayos de pulpaje bajo diferentes condiciones, variando la concentración de álcali efectivo entre 18 y $20 \%$ y el tiempo a temperatura máxima entre 30 y $60 \mathrm{~min}$, el resto de las variables permanecieron constantes.

Se obtuvieron pulpas con índices kappa entre 20 y 40, y rendimientos clasificados entre 40 y $46 \%$ respectivamente.

Palabras claves: Pulpa Kraft, Pinus patula, Pinus elliottii, Pinus taeda.

\footnotetext{
(*) Ingeniero Civil Quimico

(*) Técnico Celulosa y Papel 


\section{ABSTRACT}

The evaluation of pulping results with Pinus patula, Pinus taeda and Pinus elliottii wood grown in Chile is the objetive of this work. The wood samples were provided by the Universidad Austral de Chile from trials located at the Isla Teja campus.

Wood chemical composition and chips density were determined. Pulping processes were tryed, under different conditions, changing effective alkali concentrations from 18 to $20 \%$ and the time at maximun temperature between 30 and 60 minutes.

Pulps with Kappa indexes ranging from 20 to 40 and clasified yields between 40 to $46 \%$ were obtained.

Keywords: Kraft pulping. Pinus patula, Pinus taeda, Pinus elliottii. Chemical analysis. Chips. Pulp properties. 


\section{INTRODUCCION}

Las especies de fibra larga, destacándose entre ellas Pino insigne (Pinus radiata D. Don.) tienen una gran importancia en la industria de celulosa. Ello se refleja en su alta demanda pues, de los 37.000 .000 de metros cúbicos de madera pulpable, trozos y astillas, que se comercializan a nivel mundial, dos tercios corresponden a maderas de coniferas.

El desarrollo alcanzado por la industria de celulosa y papel en Chile, se ha basado en el monocultivo existente de pino insigne, debido a que esta especie tiene buenas aptitudes como fuente de material fibroso para la obtención de pulpa química.

Desarrollar aún más esta industria requiere de la investigación que conduzca a la búsqueda de nuevas especies que resulten atractivas como fuente de materia prima para la obtención de pulpa química. Por tal motivo resulta interesante estudiar la aptitud pulpable de las especies Pinus patula, Pinus taeda y Pinus elliottii.

Algunos antecedentes disponibles señalan que a partir de cada una de estas especies es posible obtener pulpa mediante el proceso kraft, utilizando una carga de álcali activo en el rango normal para coniferas, obteniéndose una pulpa con rendimiento promedio (FAO, 1975). Por otro lado las pulpas de Pinus elliottii y Pinus taeda, se caracterizan por tener alto factor de rasgado y baja longitud de ruptura, al ser comparadas con pulpas sulfato provenientes de otras coniferas, pero aún asi sus propiedades físico-mecánicas en general son satisfactorias para la fabricación de papeles (FAO, 1975; Uprichard Gray, 1973).

Actualmente, no existen antecedentes sobre estas especies creciendo en Chile, tanto de su aprovechamiento industrial para la fabricación de pulpa como de su caracterización física y química.

Las muestras de madera se obtuvieron como material de raleo de parcelas de entre 11 y 13 años. Este tipo de material se enmarca dentro del abastecimiento normal de madera en la industria de celulosa chilena, debido a 
que la gran parte de las materias primas provienen de faenas de raleo, efectuadas en plantaciones de pino insigne cuyas edades varían entre 11 y 14 años.

El objetivo del estudio es evaluar la aptitud pulpable de las maderas de Pinus patula, Pinus taeda y Pinus elliottii crecidas en Chile, mediante la aplicación de un ciclo de cocción kraft, variando la carga de reactivo y el tiempo a temperatura máxima.

Además, se realizó la caracterización física y química de las especies, lo que contribuyó a determinar su calidad como material pulpable. Esta caracterización consideró densidad, longitud de fibra, lignina, solubles en: etanol-tolueno, agua fría e hidróxido de sodio al $1 \%$.

MATERIAL Y METODO

Preparación y Caracterización de la Materia Prima

Las materias primas utilizadas correspondieron a árboles de raleo de una parcela en experimentación del Arboretum de la Universidad Austral de Chile. Las semillas de Pinus elliottii y Pinus taeda son procedentes de EE.UU. y las de Pinus patula de México.

Los trozos se descortezaron en forma manual y se astillaron en una unidad industrial. Las astillas se clasificaron en harnero Williams con perforaciones circulares de acuerdo a Norma Tappi T 16-ts-61. La fracción aceptada, entre $7 / 8$ " y $3 / 16 "$ ", se almacenó para su posterior procesamiento.

A una porción representativa de la fracción se le determinó su humedad y densidad. Se realizaron además, los siguientes análisis químicos, de acuerdo a la norma que se indica:

Holocelulosa

Lignina

Solubles en agua fria

Solubles en etanoltolueno
Método de Poljak

Tappi T 222-om-88

Tappi T 207-om-88

Tappi T 5-om-88 modificado. 
Solubles en hidróxido de sodio al 1\% : Tappi T 212-om-88)

Cenizas

Tappi T 15-om-88).

\section{Ensayos de Pulpaje}

Los pulpajes se realizaron en un digestor MK System de 6,5 L de capacidad, con recirculación forzada de licor y calefacción indirecta.

Con el objeto de estudiar el comportamiento de las especies frente al proceso kraft se realizaron pulpajes utilizando diferentes condiciones. Se incluyeron como variables el tiempo a temperatura máxima y la concentración de álcali efectivo, manteniendo constantes la temperatura máxima, el tiempo hasta temperatura máxima, la sulfidez y la relación licor/madera. Las condiciones de pulpaje fueron las mismas para las tres especies en estudio y se describen en la Cuadro $\mathrm{N}^{\circ} 1$.

\section{Cuadro $\mathrm{N}^{\circ} 1$}

CONDICIONES DE PULPAJE PARA Pinus elliottii, Pinus patula y Pinus taeda

Temperatura máxima $\left({ }^{\circ} \mathrm{C}\right) \quad 170$

Tiempo hasta temperatura máxima (min) $\quad 120$

Sulfidez ( $\%$ mbs) 26

Relación licor/madera $\quad 4 / 1$

\begin{tabular}{|c|c|c|}
\hline $\begin{array}{c}\text { Pulpaje } \\
\left(\mathrm{N}^{\circ}\right)\end{array}$ & $\begin{array}{c}\text { Concentración } \\
\text { de álcali } \\
(\% \text { bms })\end{array}$ & $\begin{array}{c}\text { Tiempo a } \\
\text { Temperatura } \\
(\text { min) }\end{array}$ \\
\hline 1 & 18 & 30 \\
2 & 18 & 45 \\
3 & 18 & 60 \\
4 & 20 & 30 \\
5 & 20 & 45 \\
6 & 20 & 60 \\
\hline
\end{tabular}

Las respuestas medidas fueron rendimiento clasificado, rechazos y Kappa, para la pulpa; y álcali residual y porcentaje de sólidos, para el licor negro. Las 
pulpas se refinaron en una batidora Valley según Tappi T 200-om-85. A continuación se midieron las siguientes propiedades en hojas de ensayo de 60 $\left(\mathrm{g} / \mathrm{m}^{2}\right)$, fabricadas con pulpas a diferentes grados de refinación: Densidad, Longitud de Ruptura, Factor de Rasgado y Factor de Explosión.

\section{RESULTADOS Y DISCUSION}

\section{Caracteristicas de las Especies}

En el Cuadro $\mathrm{N}^{\circ} 2$ se muestran las características físicas y químicas para las maderas de Pinus patula, Pinus elliottii y Pinus taeda.

\section{Cuadro $\mathrm{N}^{\circ} 2$}

PROPIEDADES FISICAS Y QUIMICAS DE Pinus patula, Pinus elliottii y Pinus taeda.

\begin{tabular}{|c|c|c|c|c|c|c|}
\hline Especie & $\begin{array}{c}\text { Densidad } \\
\text { verde } \\
\left(\mathrm{kg} / \mathrm{m}^{3}\right)\end{array}$ & $\begin{array}{c}\text { Longitud } \\
\text { de Fibra } \\
\text { (mm) }\end{array}$ & $\begin{array}{l}\text { Etanol } \\
\text { tolueno }\end{array}$ & $\begin{array}{l}\text { Solubles en } \\
\text { Agua } \\
\text { frla } \\
\text { (\% bms) }\end{array}$ & $\begin{array}{l}\mathrm{NaOH} \\
\text { al } 1 \%\end{array}$ & $\begin{array}{l}\text { Lignina } \\
\text { (\%bmsle) }\end{array}$ \\
\hline Pinus & 365 & 2,3 & 2,8 & 2,0 & 16,2 & 31,1 \\
\hline Pinus elliottii & 323 & 2,4 & 2,7 & 1,7 & 15,2 & 30,7 \\
\hline Pinus taeda & 365 & 2,2 & 2,2 & 1,1 & 14,1 & 31,0 \\
\hline
\end{tabular}

\% bms : Porcentaje base madera seca

\% bmsle : Porcentaje base madera seca libre de extraíbles

La densidad básica y longitud de fibra son inferiores al promedio normal para coníferas (Rydholm, 1965). Esto indicaría presencia de madera juvenil dada por la corta edad de las muestras.

Las tres especies presentan alto contenido de lignina y tienen composición similar en extraibles. El valor de solubilidad en $\mathrm{NaOH}$ al $1 \%$ puede indicar algún comportamiento en rendimiento después en el pulpaje. 


\section{Resultados de Pulpaje}

Las respuestas de los pulpajes para Pinus patula, Pinus elliottii y Pinus taeda se presentan en los Cuadros $\mathrm{N}^{\circ} \mathrm{s} 3,4$ y 5.

En general para las tres especies la elevación del tiempo a temperatura con álcali efectivo constante, produjo una disminución en el rechazo e índice Kappa. Esta misma tendencia se observó al aumentar la concentración de álcali manteniendo el tiempo a temperatura constante.

Comparando a índice kappa 30, el Pinus elliottii alcanzó rendimiento clasificado superior $(42,5 \%)$ al de Pinus patula $(41,1 \%)$ y Pinus taeda $(40,5 \%)$.

\section{Cuadro $\mathrm{N}^{\circ} 3$}

CARACTERISTICAS DE LAS PULPAS DE Pinus patula

\begin{tabular}{|c|c|c|c|c|c|c|c|}
\hline $\begin{array}{c}\text { Pulpaje } \\
\left(\mathrm{N}^{\circ}\right)\end{array}$ & $\begin{array}{c}\text { Rendim. } \\
\text { Clasif. } \\
\text { (\% bms) }\end{array}$ & $\begin{array}{c}\text { Rechazos } \\
\text { (\% bms) }\end{array}$ & $\begin{array}{c}\text { Rend. } \\
\text { Total } \\
(\% \text { bms })\end{array}$ & $\begin{array}{c}\text { Indice } \\
\text { Kappa }\end{array}$ & $\begin{array}{c}\text { Solidos } \\
\text { totales } \\
(\% \text { p/ } /)\end{array}$ & $\begin{array}{c}\text { Alcali } \\
\text { Residual } \\
(g / L)\end{array}$ & $\begin{array}{c}\text { Alcali } \\
\text { consumido } \\
(\% \text { bms })\end{array}$ \\
\hline 1 & 42,7 & 2,3 & 45,0 & 33 & 23,3 & 19,9 & 10,0 \\
$2^{*}$ & 41,7 & 3,3 & 45,0 & 31 & 24,6 & 13,4 & 12,6 \\
3 & 41,8 & 2,1 & 43,9 & 27 & 24,3 & 13,5 & 12,6 \\
4 & 39,0 & 5,2 & 44,2 & 25 & 24,1 & 24,2 & 10,3 \\
$5^{*}$ & 40,6 & 1,9 & 42,5 & 21 & 25,0 & 18,1 & 12,8 \\
6 & 40,7 & 0,6 & 41,3 & 21 & 26,1 & 17,9 & 12,8 \\
\hline
\end{tabular}

Pulpas batidas

\% bms: Porcentaje base madera seca 


\section{Cuadro $\mathrm{N}^{\circ} 4$}

CARACTERISTICAS DE LAS PULPAS DE Pinus elliottii

\begin{tabular}{|c|c|c|c|c|c|c|c|}
\hline $\begin{array}{c}\text { Pulpaje } \\
\left(\mathrm{N}^{\circ}\right)\end{array}$ & $\begin{array}{c}\text { Rendim. } \\
\text { Clasif. } \\
(\% \text { bms })\end{array}$ & $\begin{array}{c}\text { Rechazos } \\
(\% \text { bms })\end{array}$ & $\begin{array}{c}\text { Rend. } \\
\text { Total } \\
(\% \text { bms })\end{array}$ & $\begin{array}{c}\text { Indice } \\
\text { Kappa }\end{array}$ & $\begin{array}{c}\text { Solidos } \\
\text { Totales } \\
(\% \text { p/v })\end{array}$ & $\begin{array}{c}\text { Alcali } \\
\text { Residual } \\
(\mathrm{g} / \mathrm{L})\end{array}$ & $\begin{array}{c}\text { Alcali } \\
\text { Consumido } \\
(\% \mathrm{bms})\end{array}$ \\
\hline 1 & 46,3 & 0,9 & 47,2 & 47 & 21,9 & 16,9 & 11,2 \\
$2^{*}$ & 42,7 & 1,2 & 43,9 & 29 & 24,2 & 15,7 & 11,7 \\
3 & 41,1 & 0,4 & 41,5 & 19 & 24,8 & 14,2 & 12,3 \\
$4^{*}$ & 43,6 & 2,7 & 46,3 & 28 & 25,9 & 22,3 & 11,1 \\
$5^{*}$ & 41,2 & 0,4 & 41,6 & 20 & 26,3 & 19,9 & 12,0 \\
& 40,1 & 0,4 & 40,5 & 16 & 27,5 & 18,4 & 12,6 \\
\hline
\end{tabular}

Pulpas batidas

\% bms : Porcentaje base madera seca

\section{Cuadro $\mathrm{N}^{\circ} 5$}

CARACTERISTICAS DE LAS PULPAS DE Pinus taeda

\begin{tabular}{|c|c|c|c|c|c|c|c|}
\hline Pulpaje & $\begin{array}{c}\text { Rendim. } \\
\text { Clasif. } \\
(\% \mathrm{bms})\end{array}$ & $\begin{array}{c}\text { Rechazos } \\
(\% \mathrm{bms})\end{array}$ & $\begin{array}{c}\text { Rend. } \\
\text { Total } \\
(\% \mathrm{bms})\end{array}$ & $\begin{array}{c}\text { Indice } \\
\text { Kappa }\end{array}$ & $\begin{array}{c}\text { Solidos } \\
\text { Totales } \\
(\% \mathrm{p} / \mathrm{v})\end{array}$ & $\begin{array}{c}\text { Alcali } \\
\text { Residual } \\
(\mathrm{g} / \mathrm{L})\end{array}$ & $\begin{array}{c}\text { Alcali } \\
\text { Consumido } \\
(\% \mathrm{bms})\end{array}$ \\
\hline 1 & 40,9 & 4,1 & 45,0 & 38 & 23,8 & 12,3 & 13,1 \\
$2^{*}$ & 41,5 & 1,7 & 43,2 & 31 & 25,3 & 13,7 & 12,5 \\
3 & 40,1 & 1,7 & 41,8 & 26 & 23,8 & 13,4 & 12,7 \\
4 & 40,3 & 2,9 & 43,2 & 27 & 23,2 & 15,1 & 14,0 \\
$5^{*}$ & 40,3 & 0,4 & 40,7 & 22 & 25,2 & 16,2 & 13,5 \\
6 & 39,8 & 1.0 & 40,8 & 19 & 25,4 & 16,3 & 13,5 \\
\hline
\end{tabular}

\footnotetext{
- Pulpas batidas

\% bms : Porcentaje base madera seca
}

El consumo de reactivo tuvo un comportamiento diferente entre las especies. En los pulpajes con Pinus patula y Pinus elliottii los consumos aumentaron en un $25 \%$ y un $12 \%$ respectivamente, al prolongar el tiempo a temperatura máxima desde 30 a 60 minutos. En cambio con Pinus taeda el consumo de reactivo disminuyó aproximadamente un $4 \%$ al aumentar este tiempo.

En la Figura $\mathrm{N}^{\circ} 1$ se observa la relación entre el Indice Kappa obtenido y el 
tiempo a temperatura máxima aplicado en cada ciclo con álcali igual a $18 \%$ bms.

Se puede ver que en el caso de Pinus elliottii un aumento del tiempo a temperatura máxima significó una disminución notable del índice Kappa en las pulpas, alcanzándose una reducción en este índice de un $50 \%$ entre 30 y 60 minutos. En cambio para Pinus taeda y Pinus patula el kappa decreció en un $30 \%$ y un $18 \%$, respectivamente. Cabe notar que con esta última especie se obtuvieron porcentajes de rechazo superiores en todos los pulpajes. Esto indica que la penetración del reactivo hacia el centro de las astillas no fue completa.

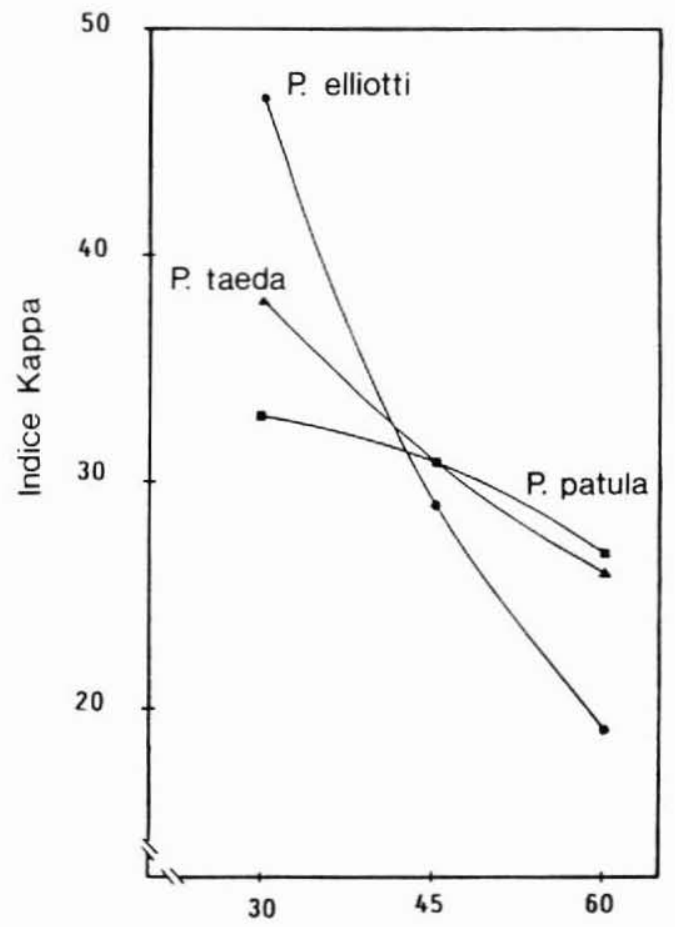

Tiempo a tem. máx. (min.)

Figura $N^{\circ}$ 1. RELACION ENTRE INDICE KAPPA Y EL TIEMPO A TEMPERATURA MAXIMA (ALCALI ACTIVO: $18 \% \mathrm{bms}$ ) 


\section{Propiedades Físico-Mecánicas de las Pulpas}

Para el análisis de las propiedades físico-mecánicas se eligieron las pulpas provenientes de los pulpajes 2 y 5 por tener índices kappa cercanos a 30 y 20 , respectivamente. El kappa igual a 30 se eligió por ser característico de las pulpas de coníferas obtenidas a nivel industrial, por otro lado el kappa igual 20 se seleccionó para comparar el comportamiento de las pulpas a un contenido menor de lignina. Del análisis de estas pulpas se pretende además, ver la influencia de la concentración de álcali activo sobre las propiedades finales de la pulpa.

A continuación se indican los resultados del análisis de las pulpas por especie.

\section{Pinus patula}

En los Cuadros $N^{\circ}$ s 6 y 7 se presentan las propiedades físico-mecánicas de las pulpas de Pinus patula, refinadas a diferentes grados Schopper.

\section{Cuadro $\mathrm{N}^{\circ} 6$ \\ PROPIEDADES DE LAS PULPAS DE Pinus patula \\ Pulpaje $N^{\circ} 2$}

\begin{tabular}{|c|c|c|c|c|c|}
\hline Tiempo & Drenaje & Densidad & $\begin{array}{c}\text { Long. } \\
\text { ruptura } \\
(\mathrm{km})\end{array}$ & $\begin{array}{c}\text { Factor } \\
\text { explosión } \\
(-)\end{array}$ & $\begin{array}{c}\text { Factor } \\
\text { rasgado } \\
(\mathrm{min})\end{array}$ \\
\hline 0 & $\left({ }^{\circ} \mathrm{SR}\right)$ & $\left(\mathrm{g} / \mathrm{cm}^{3}\right)$ & 68 & 79 \\
5 & 15,0 & 0,600 & 6,87 & 55 & 71 \\
18 & 17,0 & 0,693 & 8,69 & 65 & 55 \\
24 & 25,0 & 0,760 & 10,30 & 73 & 52 \\
29 & 35,0 & 0,805 & 11,05 & 77 & 50 \\
\hline
\end{tabular}




\section{Cuadro $\mathrm{N}^{\circ} 7$}

PROPIEDADES DE LAS PULPAS DE Pinus patula Pulpaje $\mathbf{N}^{\circ} \mathbf{G}$

\begin{tabular}{|c|c|c|c|c|c|}
\hline Tiempo & Drenaje & Densidad & $\begin{array}{c}\text { Long. } \\
\text { ruptura } \\
(\mathrm{km})\end{array}$ & $\begin{array}{c}\text { Factor } \\
\text { explosión } \\
(-)\end{array}$ & $\begin{array}{c}\text { Factor } \\
\text { rasgado } \\
(\mathrm{min})\end{array}$ \\
\hline 0 & $\left({ }^{\circ} \mathrm{SR}\right)$ & $\left(\mathrm{g} / \mathrm{cm}^{3}\right)$ & 29 & 87 \\
5 & 15,5 & 0,603 & 6,55 & 58 & 72 \\
21 & 16,5 & 0,692 & 8,70 & 75 & 62 \\
28 & 25,0 & 0,788 & 10,30 & 78 & 58 \\
32 & 35,0 & 0,818 & 10,80 & 77 & 55 \\
\hline
\end{tabular}

La pulpa $\mathrm{N}^{\circ} 2$ (Kappa igual a 31 ) requirió menor tiempo de batido para alcanzar un mismo grado de refinación que la pulpa $\mathrm{N}^{\circ} 5$ (Kappa igual a 21).

No hubo diferencias apreciables en densidad entre las pulpas $N^{\circ} \mathrm{s} 2$ y 5 ; esto significa que en la pulpa $\mathrm{N}^{\circ} 2$ el espesor de pared no se vio aumentado por el mayor contenido de lignina en esta pulpa. La longitud de ruptura registró un valor levemente superior en la pulpa $\mathrm{N}^{\circ} 2$

La pulpa $\mathrm{N}^{\circ} 2$ tuvo menor factor de rasgado, lo cual se explica por los pentosanos presentes en la pulpa que influyen en los enlaces interfibra mejorando levemente la longitud de ruptura. La resistencia al rasgado en general varía inversamente con la cantidad de enlaces interfibra.

Si se comparan ambas pulpas a Schopper inicial y a $45^{\circ} \mathrm{SR}$, en donde la densidad es prácticamente la misma, se puede ver además que el factor de explosión en estos puntos tampoco muestra diferencias apreciables. Lo anterior muestra que existiria una relación entre la densidad del papel y el factor de explosión.

\section{Pinus elliottii}

En los Cuadros $\mathrm{N}^{\circ} \mathrm{s} 8$ y 9 se presentan las propiedades físico-mecánicas de las pulpas de Pinus elliotti. 


\section{Cuadro $\mathrm{N}^{\circ} 8$}

PROPIEDADES DE LAS PULPAS DE Pinus elliotti Pulpaje $N^{\circ} 2$

\begin{tabular}{|c|c|c|c|c|c|}
\hline Tiempo & Drenaje & Densidad & $\begin{array}{c}\text { Long. } \\
\text { ruptura } \\
(\mathrm{km})\end{array}$ & $\begin{array}{c}\text { Factor } \\
\text { explosion } \\
(-)\end{array}$ & $\begin{array}{c}\text { Factor } \\
\text { rasgado } \\
(\mathrm{min})\end{array}$ \\
\hline 0 & $\left({ }^{\circ} \mathrm{SR}\right)$ & $\left(\mathrm{g} / \mathrm{cm}^{3}\right)$ & 6,02 & 64 & 122 \\
5 & 16.0 & 0,517 & 6,02 & 83 \\
36 & 17,0 & 0,642 & 8,75 & 83 & 67 \\
48 & 25,0 & 0,777 & 10,60 & 86 & 60 \\
55 & 35,0 & 0,810 & 11,20 & 89 & 57 \\
\hline
\end{tabular}

Cuadro $\mathrm{N}^{\circ} \mathrm{g}$

PROPIEDADES DE LAS PULPAS DE Pinus elliotti Pulpaje $N^{\circ} 5$

\begin{tabular}{|c|c|c|c|c|c|}
\hline $\begin{array}{l}\text { Tiempo } \\
(\mathrm{min})\end{array}$ & $\begin{array}{c}\text { Drenaje } \\
\text { ('SR) }\end{array}$ & $\begin{array}{l}\text { Densidad } \\
\left(\mathrm{g} / \mathrm{cm}^{3}\right)\end{array}$ & $\begin{array}{l}\text { Long. } \\
\text { ruptura } \\
(\mathrm{km})\end{array}$ & $\begin{array}{c}\text { Factor } \\
\text { explosión } \\
(-)\end{array}$ & $\begin{array}{c}\text { Factor } \\
\text { rasgado } \\
(-)\end{array}$ \\
\hline 0 & 16,0 & 0,574 & 7,51 & 56 & 103 \\
\hline 5 & 17,0 & 0,675 & 8,83 & 64 & 83 \\
\hline 30 & 25,0 & 0,785 & 11,90 & 80 & 65 \\
\hline 41 & 350 & 0.815 & 12.20 & 87 & 57 \\
\hline 46 & 45,0 & 0,828 & 11,55 & 90 & 54 \\
\hline
\end{tabular}

Con la pulpa $\mathrm{N}^{\circ} 5$ se obtuvieron factores de explosión y rasgado menores lo que indica que hubo pérdida de resistencia en el proceso de pulpaje producto de la mayor concentración de reactivo. Por otro lado la longitud de ruptura es mayor para esta pulpa indicando una mayor unión entre fibras, lo cual está dado también por una mayor densidad de hoja.

El tiempo de batido es menor para la pulpa $\mathrm{N}^{\circ} 5$ con menor contenido de lignina (kappa igual a 20) resultando en mayor facilidad al hinchamiento en el proceso de la refinación. La unión fibrilar también depende de la fibrilación producto de la refinación y al existir ésta, se alteran las dimensiones de las fibras lo que hace el que el factor de rasgado disminuya. 


\section{Pinus taeda}

En los Cuadros $\mathrm{N}^{\circ} \mathrm{s} 10$ y 11 se presentan las propiedades físico-mecánicas de las pulpas de Pinus taeda.

\section{Cuadro $\mathrm{N}^{\circ} 10$}

PROPIEDADES DE LAS PULPAS DE Pinus taeda

Pulpaje $N^{\circ} 2$

\begin{tabular}{|c|c|c|c|c|c|}
\hline $\begin{array}{l}\text { Tiempo } \\
\text { (min) }\end{array}$ & $\begin{array}{c}\text { Drenaje } \\
\text { ('SR) }\end{array}$ & $\begin{array}{l}\text { Densidad } \\
\left(\mathrm{g} / \mathrm{cm}^{3}\right)\end{array}$ & $\begin{array}{l}\text { Long. } \\
\text { ruptura } \\
\text { (km) }\end{array}$ & $\begin{array}{c}\text { Factor } \\
\text { explosión } \\
(-)\end{array}$ & $\begin{array}{c}\text { Factor } \\
\text { rasgado } \\
(-)\end{array}$ \\
\hline 0 & 15,5 & 0,601 & 6,66 & 57 & 111 \\
\hline 5 & 16,5 & 0,718 & 8,52 & 70 & 80 \\
\hline 20 & 25,0 & 0,770 & 9,80 & 79 & 75 \\
\hline 30 & 35,0 & 0,820 & 11,25 & 82 & 66 \\
\hline 35 & 45,0 & 0,843 & 12,15 & 80 & 59 \\
\hline
\end{tabular}

\section{Cuadro $\mathrm{N}^{\circ} 11$}

PROPIEDADES DE LAS PULPAS DE Pinus taeda Pulpaje $\mathbf{N}^{\circ} 5$

\begin{tabular}{|c|c|c|c|c|c|}
\hline $\begin{array}{l}\text { Tiempo } \\
\text { (min) }\end{array}$ & $\begin{array}{c}\text { Drenaje } \\
\text { (SR) }\end{array}$ & $\begin{array}{c}\text { Densidad } \\
\left(\mathrm{g} / \mathrm{cm}^{3}\right)\end{array}$ & $\begin{array}{l}\text { Long. } \\
\text { ruptura } \\
\text { (km) }\end{array}$ & $\begin{array}{c}\text { Factor } \\
\text { explosión } \\
(-)\end{array}$ & $\begin{array}{c}\text { Factor } \\
\text { rasgado } \\
(-)\end{array}$ \\
\hline 0 & 17,0 & 0,584 & 6,59 & 56 & 128 \\
\hline 5 & 18,0 & 0,725 & 8,68 & 59 & 95 \\
\hline 23 & 25,0 & 0,795 & 10,65 & 77 & 76 \\
\hline 32 & 35,0 & 0,835 & 11,55 & 86 & 65 \\
\hline 37 & 45,0 & 0,845 & 11,75 & 90 & 58 \\
\hline
\end{tabular}

El tiempo de batido es similar entre las pulpas $\mathrm{N}^{\circ} 2$ y $\mathrm{N}^{\circ} 5$ (Kappa igual a 31 y 22, respectivamente). A Schopper inicial la pulpa $\mathrm{N}^{\circ} 2$ alcanzó mayor densidad de hoja producto de mejor compactación, pero a medida que se bate. ésta se hace menor. 
En los primeros puntos el factor de rasgado es mayor en la pulpa con menor contenido de lignina, pero entre 25 y $45^{\circ} \mathrm{SR}$ no se aprecian diferencias. La longitud de ruptura es levemente superior en la pulpa $\mathrm{N}^{\circ} 5$ entre 18 y $35^{\circ} \mathrm{SR}$. El factor de rasgado hasta $25^{\circ} \mathrm{SR}$ es mayor en la pulpa $\mathrm{N}^{\circ} 2$, luego de este punto se hace menor en esta pulpa.

\section{CONCLUSIONES}

La composición química de las especies Pinus patula, Pinus elliottii y Pinus taeda no implicó ninguna dificultad en el pulpaje.

A indice Kappa 30 Pinus elliotti alcanzó un rendimiento clasificado levemente superior $(42,5 \%)$ a los de Pinus patula $(41,1 \%)$ y Pinus taeda $(40,5 \%)$.

Las pulpas de Pinus patula requirieron tiempos de batido menores que Pinus taeda y Pinus elliottii para un mismo grado de refinación.

Pinus elliottii y Pinus taeda tuvieron alto factor de rasgado. La longitud de ruptura y factor de explosión fue similar en las tres especies.

En general, las pulpas de Pinus patula, Pinus taeda y Pinus elliottii tienen buenas características para la fabricación de papeles.

\section{REFERENCIAS BIBLIOGRAFICAS}

FAO., 1980. Pulping and Paper-Making Properties of Fast-Growing Plantation. Wood Species Vol. 1. Rome 347, 383, 411 p.

Rydholm, S., 1965. Pulping Processes. New York. Interscience Publishers.

Uprichard, J. and Gray, J., 1973. Papermaking Properties of Kraft Pulp from New Zealand Grown Softwoods. Appita Vol. 27(3) 185: 191. 